\title{
25
}

\section{Human Modelling and Design of Spatio-Temporal Queries on 3D Video Database}

Takashi Tomii, Kiril Salev, Sayaka Imai and Hiroshi Arisawa Division of Electrical and Computer Engineering

Faculty of Engineering

Yokohama National University

79-5 Tokiwadai, Hodogaya-ku, Yokohama, 240-0067 JAPAN

Phone:+81-45-339-4160 Fax:+81-45-338-1012

E-mail:\{tommy,kitcho,sayaka,arisawa\}@arislab.dnj.ynu.ac.jp

\begin{abstract}
This paper presents a concept of a 3D Video Database, called Real World Database (RWDB), in which not only image and audio data but also object structure, behavior, temporal event and all spatial occasions are stored uniformly. In this presentation, we will concentrate ourselves into the factory workers' modelling and simulation for the virtual manufacturing systems. Using RWDB, we designed the human body structures, motions in a work and events over them. As a result, it can evaluate various motions of workers and pursue the optimum design of works. Also a functional data manipulation language MMQL for spatio-temporal inquiry is offered. This enables us to evaluate the posture and safety of workers based on the calculation of positions of spatial objects.
\end{abstract}

\section{Keywords}

Spatio-Temporal Database, 3D Image Processing, Spatio-Temporal Query, Human Body Modelling, Factory Worker Simulation, Info-Ergonomics

\section{INTRODUCTION}

With the recent advancement of computer technology, easy handling of visual multimedia data like computer graphics (CG), images and video became possible. In particular, high performance computers and image and graphic data processing technology led to the creation of many virtual reality (VR) systems which allow realistic representation of the virtual world (Hirose, M. 1997, Kanade, T. et al. 1997). Construction of a realistic virtual space has been widely discussed and many proposals have been made about the modelling of temporal and spatial objects in the real world and their stor- 
ing, maintenance, and retrieval methods. We call spatio-temporal database a database that can store informations about objects and events in the real world and achieve subsequent retrieving of their position and movements.

Typical problems when considering spatio-temporal databases are the capturing and analysis of 3-dimensional (3D) data, time and event modelling approaches, requirements to analyse the underlying data model and manipulation language. 3D CAD systems and geographical databases are dealing with space and time modelling but they provide only partial solutions to some specific problems. Also, as those applications use mainly applicationoriented data, data sharing/integration among different applications is quite difficult and very often impossible - widely adopted methodology for capturing, modelling, and manipulating spatio-temporal data does not exist yet.

As a promising solution to the above problems we have offered the use of Real World Database (RWDB) (Arisawa, H. 1996). It is capable of capturing various types of data from video images to $3 \mathrm{D}$ polygons. The data of all types are stored into a multimedia database in a uniform way. An important characteristic of the RWDB is that all data are stored as they are observed, without any compression or transformation, enabling the realistic reorganization of the virtural world using a given media.

Based on the information captured from RWDB, observed objects and events can be precisely visualized by using high-resolution computer graphics, thus creating a virtual space. The methodology we offer allows us not only to query the objects' position and movements, but also to perform simulations based on the real world data.

Possible applications of the proposed approach include factory work planing and work estimation, sports, medicine, rehabilitation, etc. As humans are playing an important role in all those fields, we consider in more detail here the human modelling.

At first, we introduce the concept of RWDB in next section. Section 3 discusses about modelling methodology of human body and events in RWDB, and in section 4, the framework of data model AIS (Arisawa, H. et al. 1995) and the way to represent 3D objects and motions are discussed. Section 5 shows a query language MMQL (Arisawa, H. et al. 1996) for spatio-temporal queries.

\section{REAL WORLD DATABASE}

The objective of the RWDB is to provide a total environment of capturing, modelling and storing physical or logical objects from the real world. Everything in the real world could be modelled through it and any types of data could be accumulated. For this purpose, RWDB must involve at least 4 components listed below. Conceptual architecture of RWDB is shown in Figure 1. 


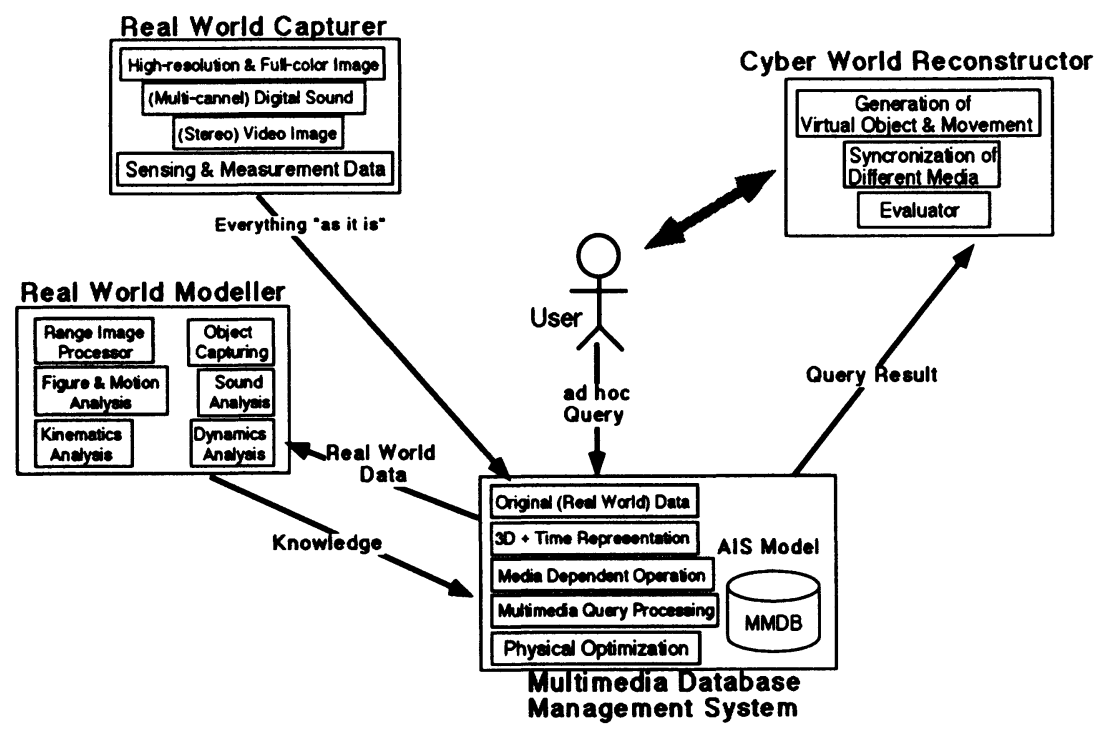

Figure 1 Conceptual architecture of RWDB

\section{- Real World Capturer (RWC)}

The objective of RWC is to capture the external form of objects in the real world. There exist various types of $3 D$ or spatial information from the real world depending on capturing devices. The simplest one is a stereo pair of images by video cameras. We can get a sequence of frames from, for example, a left-eye camera and a right one simultaneously. Recent technology enables us to get the full-color and high quality digital image with no compression (i.e. lossless) of data. However, for the frame sequences, it is very hard to extract a complete surface model or motion (kinematics) model of each object. On the other hand, several "Motion Capturing Systems" are commercially available but those systems can trace only a small number of marking points and considerable part of original information (surface and texture) is lost.

Another type of input device is a "3D Scanner" with which we can get a complete surface (polygon) model for static objects. The practical solution is to get the above two kinds of information from the real world and to combine the two models into one at the database level. This idea is summarized in Figure 2.

- Real World Modeller (RWM)

RWM is a set of tools each of which analyzes original frame images and generates new information. For example, the "outline chaser" catches the outline of an object in a certain video frame, and then traces the outline in preceding and successive frames. On the other hand, the "point tracer" detects stereo pairs and calculates the range values for (specified) points 


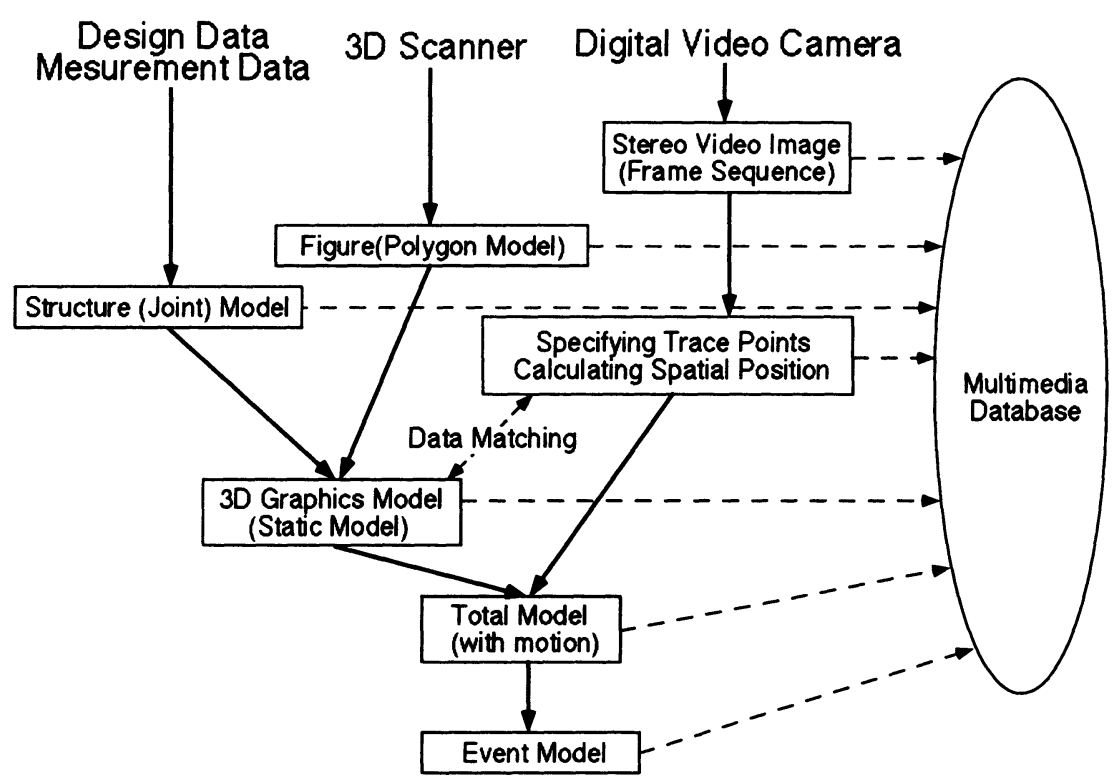

Figure 2 Integration of various types of 3D Data

from a couple of (left and right) frame images, and makes a rough sketch of $3 \mathrm{D}$ objects in the real world. Many algorithms are investigated and evaluated for range image generation in the image processing area (Arisawa, H. et al. 1995). All the results of the analysis are stored into a database preserving the correspondences to the original images.

- Multimedia Database (MMDB)

MMDB is a database that treats a variety of data types such as full text, graphic drawings, bitmap images and image sequences. The features of such data are quite different from the conventional DBMS's ones, because some of them are continuous and might occupy much more space than traditional data types. As to data model, in order to integrate all types of data, introduction of simple primitives to describe real world entities and the associations between them are essential. Moreover, the query language for handling multimedia data must involve various types of media-dependent operations for retrieving and displaying. Especially, in RWDB, the result of a query creates a new "cyberspace" for intuitive understanding. The authors proposed a total data model to describe the 2D or 3D data, and also presented query language MMQL for flexible retrieval (Arisawa, H. et al. 1995) which is introduced in later sections.

- Cyber World Reconstructor (CWR)

As discussed above, the result of an inquiry consists of various types of data such as frame sequences and 3D graphics data. In order to visualize the query result, RWDB should provide a player (viewer) of the result world. 


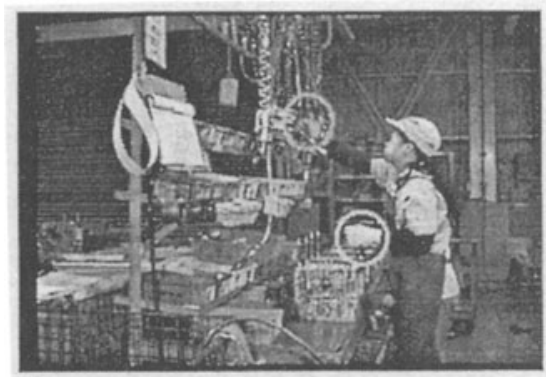

Left Image

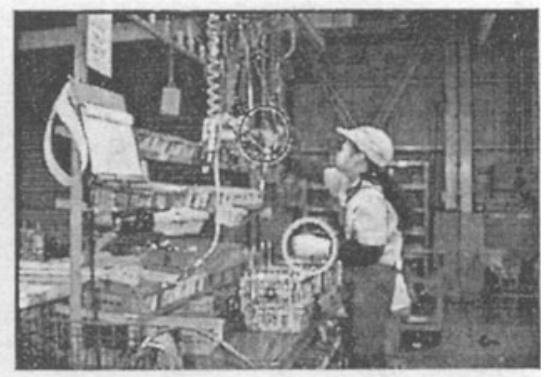

Right Image

Figure 3 example images of a stereo pair

CWR is, in this sense, an integrated system of 3D computer graphics and $3 \mathrm{D}$ video presentation.

Unfortunately, the modelling methods of objects in the field of 3D graphics and VR systems are quite different from the DB approach because the former are focusing on natural and smooth presentation of object surfaces and their motions, whereas the latter requires deep considerations on the semantic aspects of real world objects.

\section{MODELLING OF HUMANS AND EVENTS}

Based on the above RWDB concept, we concentrate efforts on the modelling of human bodies and motions because the human body is a most popular "structual" object in the real world and the analysis of its motion can be applied to a number of application fields. Among them, in our current work, we are focusing on human-machine co-operative work in factory automation. We offered the human working model for analyzing motions and reconstructing human working in CG simulation. For example, for a factory work database which includes unit works of human workers, RWM might extract a worker's motion in each work. A simple example of capturing a worker's motion is shown in Figure 3. It shows the result of detection of the right and left hands at a point of time. The spatial positions of both hands are easily calculated by using stereo images.

Also the factory itself is stored in the database as cyberspace by using CG like polygon model. A database query may retrieve a certain unit work and project it to another human worker. For such purposes, we offered InfoErgonomics (Arisawa, $\mathrm{H}$ et al. 1996) which modells human structure, motions and contents of motions, and can apply these data for product application. 


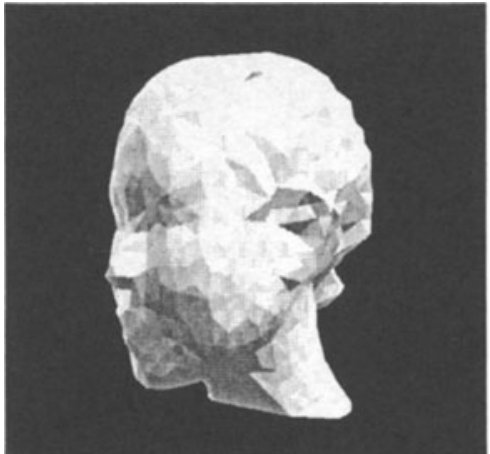

(a) Polygon Model

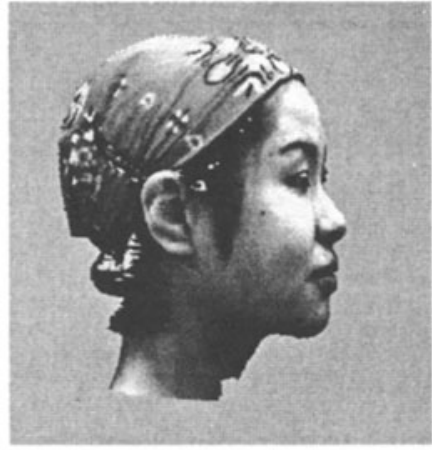

(b) Texture Image

Figure 4 Polygon Model and Texture Image of a Head

\subsection{Human Model based on Info-Ergonomics}

In order to simulate the factory employee's work, we need an integrated data model which can describe all kind of objects and materials in the factory, including the human body, for use in all applications. Creating a realistic model of the human body requires a considerable amount of data because of the object's complexity. Therefore from an ergonomical point of view we must re-model the human body with a small number of primitives. We call it the "Simplified Human Body", which involves, for example, simplified head, body, arms and legs, connected by a small number of joints.

There are two phases in based modelling on Info-Ergonomics.

\section{(a) Human Static Model}

\section{Polygon Model}

The human body's figure can be described by using polygon and texture models. An example of the polygon model alone, consisting of 1,500 polygons, is shown in Figure $4(\mathrm{a})$, and a complete texture image is shown in Figure 4 (b).

2. Structure Model

The human body consists of more than 200 bones, which are connected so that the body can move using the power of the muscles. But that mechanism is too complex to model it completely. We focus only on the movable joints and select. 24 of them in order to create a simplified model of the human body - the Human Skeleton Model. Each child component of the Human Skeleton Model has its own coordinate system, the origin of which is the connecting point (i.e. joint) to the parent component.

An example of a Human Skeleton Model and connections between parts are shown in Figure 5. 


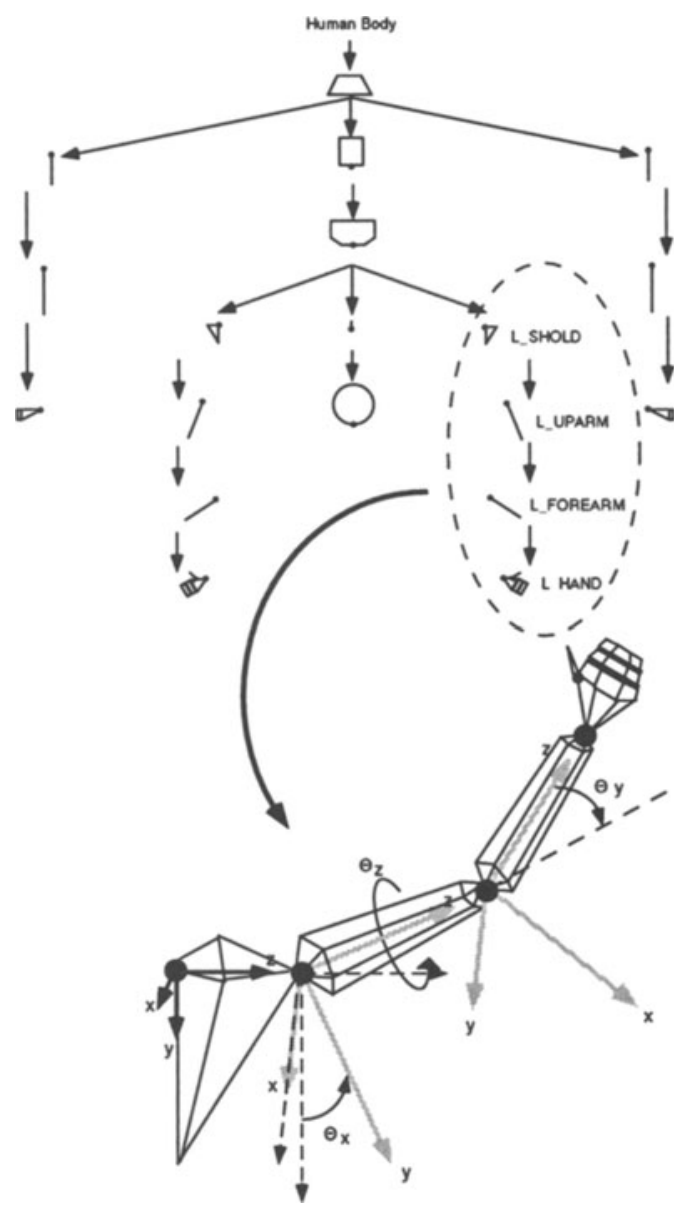

Figure 5 Structure Model

Table 1 shows an example of such parent-child relationships using a relational database-like style. For each child component, the parent component, the position $(x, y, z)$ of joint and initial angles $\left(\theta_{x}, \theta_{y}, \theta_{z}\right)$ to the parent are saved.

Actual movements of the joints have strong connections with the muscles. In our research an additional simplification of the considerations is achieved by considering anly the range of motion for the selected 24 joints. Each joint has a degree of freedom less than three, rotation or translation. In Figure 5, the joints expressed by cycle can rotate. We can do this because it would be possible to compare the employee's work with the machine's work, especially regarding to their abilities to perform certain actions, if we regard the human body as a "rigid" object. 
Table 1 Tabular Representation of Human Body Structure

\begin{tabular}{llrrrrrr}
\hline \multirow{2}{*}{ Name } & \multirow{2}{*}{ Parent } & \multicolumn{1}{c}{ Relative Position(mm) } & \multicolumn{3}{c}{ Initial Angle(degrees) } \\
\cline { 2 - 8 } Base & - & 0.00 & 0.00 & 0.00 & 0.00 & 0.00 & 0.00 \\
\hline LOIN & Base & 0.00 & 0.00 & 881.1 & 0.00 & 0.00 & 0.00 \\
\hline TORSO & LOIN & 0.00 & 0.00 & 15.00 & 0.00 & 0.00 & 0.00 \\
\hline CHEST & TORSO & 0.00 & 0.00 & 200.00 & 0.00 & 0.00 & 0.00 \\
\hline NECK & CHEST & 0.00 & 0.00 & 157.20 & 0.00 & 0.00 & 0.00 \\
\hline HEAD & NECK & 0.00 & 0.00 & 65.50 & 0.00 & 0.00 & 0.00 \\
\hline L_SHOULDER & CHEST & 0.00 & 65.00 & 207.20 & 0.00 & 0.00 & 0.00 \\
\hline L_UPARM & L_SHOULDER & 0.00 & 1044.80 & 0.00 & -180.00 & 0.00 & 0.00 \\
\hline L_FOREARM & L_UPARM & 0.00 & 0.00 & 331.50 & 0.00 & 0.00 & 0.00 \\
\hline L_HAND & L_FOREARM & 0.00 & 0.00 & 269.20 & 0.00 & 0.00 & 0.00 \\
\hline R_SHOULDER & CHEST & 0.00 & -65.00 & 207.20 & 0.00 & 0.00 & 0.00 \\
\hline R_UPARM & R_SHOULDER & 0.00 & 1044.80 & 0.00 & -180.00 & 0.00 & 0.00 \\
\hline R_FOREARM & R_UPARM & 0.00 & 0.00 & 331.50 & 0.00 & 0.00 & 0.00 \\
\hline R_HAND & R_FOREARM & 0.00 & 0.00 & 269.20 & 0.00 & 0.00 & 0.00 \\
\hline L_THIGH & LOIN & 0.00 & 81.00 & 0.00 & 180.00 & 0.00 & 0.00 \\
\hline L_LEG & L_THIGH & 0.00 & 0.00 & 377.10 & 0.00 & 0.00 & 0.00 \\
\hline L_FOOT & L_LEG & 0.00 & 0.00 & 43.34 & 0.00 & 0.00 & -30.00 \\
\hline R_THIGH & LOIN & 0.00 & -81.00 & 0.00 & 180.00 & 0.00 & 0.00 \\
\hline R_LEG & R_THIGH & 0.00 & 0.00 & 377.10 & 0.00 & 0.00 & 0.00 \\
\hline R_FOOT & R_LEG & 0.00 & 0.00 & 43.34 & 0.00 & 0.00 & 30.00 \\
\hline & & & & & & & \\
\hline
\end{tabular}

\section{(b) Human Dynamic Model}

In the Dynamic Model, human motions are described. For a certain employee's work, the action of each component of the human body should be traced and modelled under the restriction of its inter-joint structure. This Dynamic Model is defined based on the Human Skeleton Model discussed above. Each joint moves along with time. We can also use a relational database to express the joint movement as a successive relative angles every child component. An example of movement of Table 1's object, following to Table 2. By calculating the data, CG polygons of each point of time can be obtained. 
Table 2 An example of Joint Value of Work

\begin{tabular}{crrrrrr}
\hline \multirow{2}{*}{ Time } & \multicolumn{7}{c}{ INSTANCE_WORKER001 } \\
\cline { 2 - 8 } & \multicolumn{2}{c}{ L_SHOULDER001 } & \multicolumn{3}{c}{ L_UPARIM001 } \\
& $\Theta_{x}(\mathrm{deg})$ & $\Theta_{y}(\mathrm{deg})$ & $\Theta_{z}(\mathrm{deg})$ & $\Theta_{x}(\mathrm{deg})$ & $\Theta_{y}(\mathrm{deg})$ & $\Theta_{z}(\mathrm{deg})$ \\
\hline 0.00 & 4.286 & 3.249 & 0.000 & 32.57 & 0.00 & $\cdots$ \\
\hline $0: 10$ & 4.286 & 3.249 & 0.000 & 32.57 & 0.00 & $\cdots$ \\
\hline $0: 20$ & 4.286 & 3.249 & 0.000 & 32.57 & 0.00 & $\cdots$ \\
\hline $0: 30$ & 4.286 & 3.249 & 0.000 & 32.57 & 0.00 & $\cdots$ \\
\hline $0: 40$ & 5.265 & 4.228 & 0.000 & 32.57 & 0.00 & $\cdots$ \\
\hline $0: 50$ & 5.286 & 4.249 & 0.000 & 32.57 & 0.00 & $\cdots$ \\
\hline $0: 60$ & 5.332 & 4.295 & 0.000 & 32.57 & 0.00 & $\cdots$ \\
\hline $1: 00$ & 5.846 & 4.809 & 0.000 & 32.57 & 0.00 & $\cdots$ \\
\hline $1: 10$ & 6.286 & 5.249 & 0.000 & 32.57 & 0.00 & $\cdots$ \\
\hline$\cdots$ & $\cdots$ & $\cdots$ & $\cdots$ & $\cdots$ & $\cdots$ & $\cdots$
\end{tabular}

\subsection{Event Model}

When the motions of the Human Model are described, the meanings of motions or their semantics, for example, driving in a nail, lifting an object, writing characters, etc have to be discussed. We call an event everything that occurs, any significant activity during the production process.

When considering events, depending on the user's objectives, different levels of abstraction are applied. For instance, we can consider as an event simply the screwing of a screw or the making of a whole car. In the field of Industrial Engineering such separation is strictly regulated. The same production process is regarded at four different levels - production line, processes, elementary operations, and elementary movements, and there are hierarchical relations between them. An example is given, in Figure 6. Thus, events should be defined in a way that reflects such multilevel organization. The data model should provide simple and natural mappings of the hierarchical network onto the data model primitives.

\section{CONCEPTUAL SCHEMA OF THE REAL WORLD}

\subsection{The Data Model}

In this section, we will briefly discuss the data model and how to organize the multimedia data like human bodies and the texture. For intuitive understanding, we introduce a new data diagram, called AIS diagram, in order to repre- 


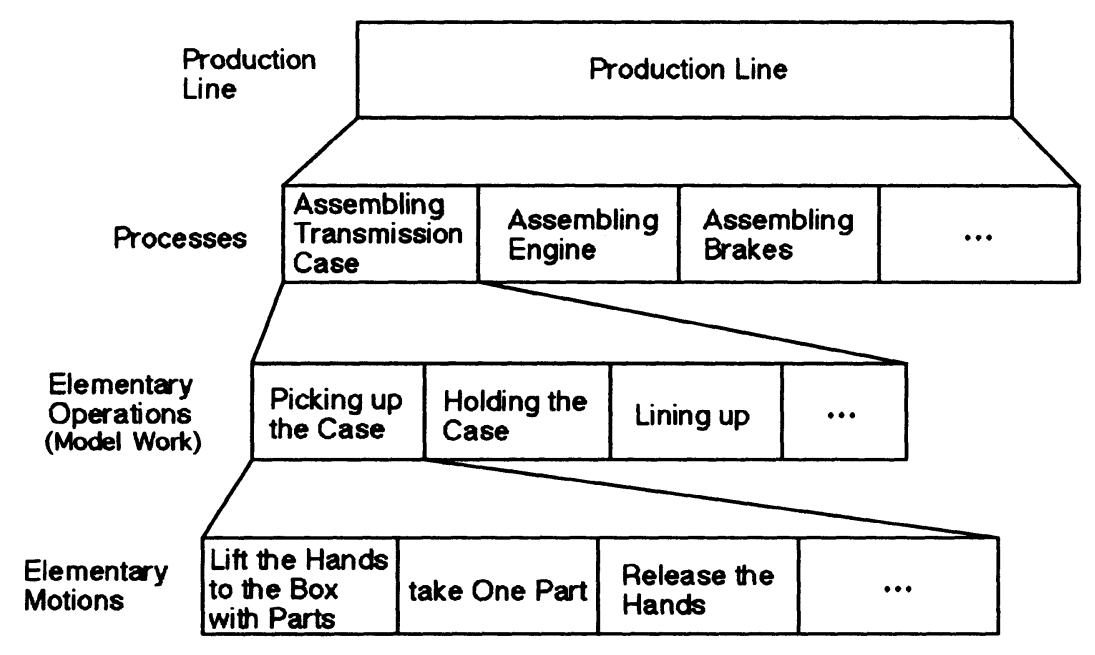

Figure 6 An Example of Event Model

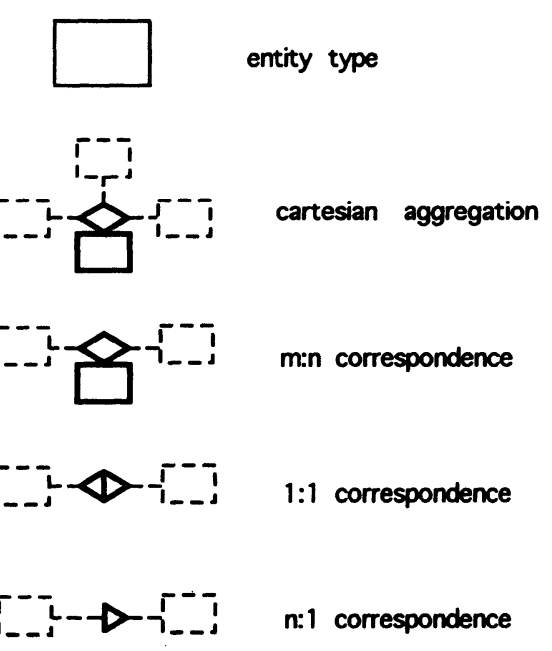

Figure 7 Design Primitives of AIS Diagram

sent information structures in multimedia applications. The basic idea of AIS (Abstract Information Structure) was presented in (Arisawa, H. et al. 1995). The AIS diagram, an extension of Chen's ER diagram (Chen, P.P. 1976), is a notation of information structure of the real world. The design primitives of AIS diagram are summarized in Figure 7.

The primitives of AIS are essentially entities and associations. AIS databases are built by these simple primitives, and, in this sense, have a "flat" structure. 
We consider that an entity is a logical object corresponding to a distinguishable "thing" in the real world, that is, entities are surrogates of real world objects. For instance, a person, a body, a part of the body, and their names are all entities. The values such as integers, real numbers, bit strings and so on are also entities, called value entities.

On the other hand, we can define a binary relation between two entity sets, and the instance of it is called an association. The association is denoted by a function enumerating instances such like $f(a)=b$, where $a$ and $b$ are entities. Note that the function may have multi-values, that is, the mapping $f(a 1)=$ $\{b 1, b 2\}$ can exist. We assume that the function is bi-directional and a oneto-many function always preserves the order of return values. We restrict the cardinalities of functions to $1: 1,1: n$ and $n: 1$ because an m:n correspondence can be divided into two 1:n mappings, and an instance of the correspondence itself can be regarded as an entity, so called a relationship entity. Formally, a relationship entity is an entity that represents an element of the Cartesian product of given entity sets.

Moreover, association is used for the definition of the component entity of an entity. For example, a part of a machine is a component that can exist under the existence of the machine. As the relationship entity is an entity, it can own component entities.

The association for component definition may be a $1: n$ function and, following the above definition, component entities of an entity always preserve certain order. For example, a scene captured by a video camera is a series of "frame" entities each of which involves a color pixel map. This extension is essential for multimedia data modelling, because series of composite objects (for example, series of "body parts") are quite popular and many media-dependent operations are applied along the sequence.

All these entities are grouped into entity types similar to ER model. The AIS diagram describes all entity types, relationship entity types and component entity types graphically. We denote an entity type, like in ER diagrams, by a rectangle, and an association between types by a line. The relationship types are denoted by lozenges or triangles depending upon the mapping cardinality between entities.

By using the association, we can define database functions by way of some entity types. The database function must be defined by enumerating all entity types on the path with the delimiter "."(dot).

\subsection{Description of Working Human Model in Database Schema}

In the database, many types of data are stored, such as image sequences, contents of work, worker's characteristics (structure and motions), and work motions. In RWDB, we consider 3-dimensional and time-spatial information 


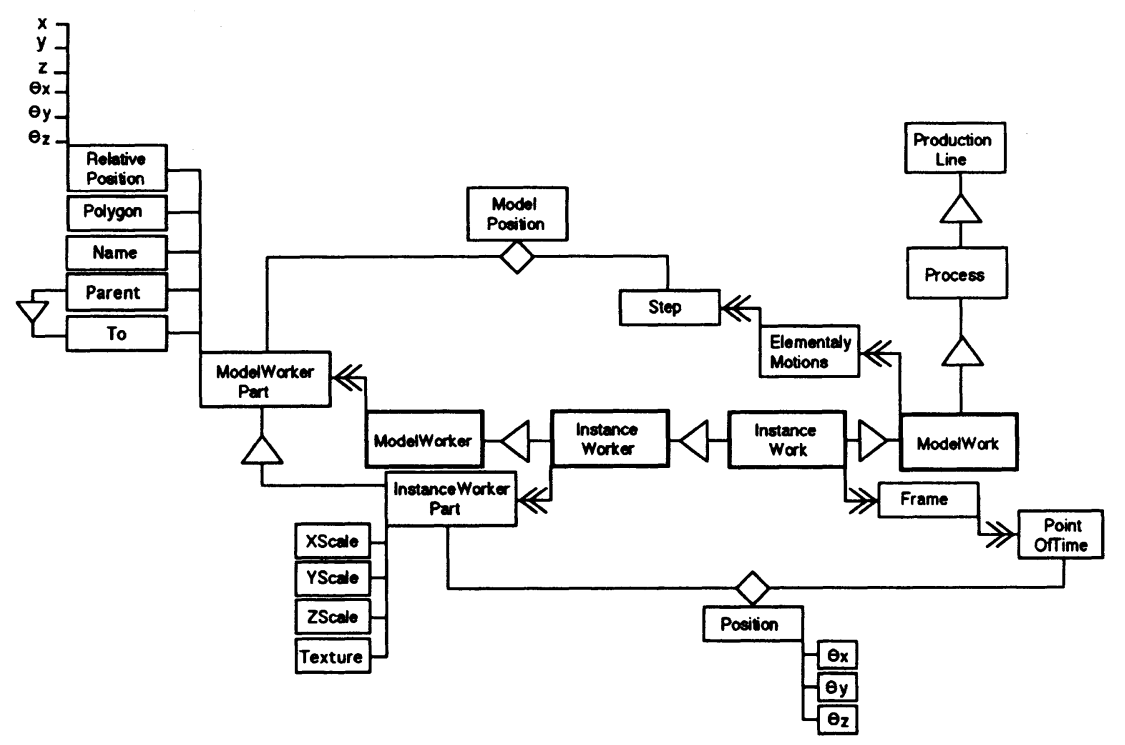

Figure 8 Schema of Human Works

from two points of view, namely data changing along with time and a structure of objects. The former represents worker's motion and position at each point of time. And the latter is common data for each object, for example, a human worker, a working machine, or a product. And the common data are regarded as knowledge about the structure. For example, when a worker model is described by a hierarchical structure as in Figure 5, each part has information about the joint position, range of motion and standard surface by polygons. Then, the whole structure is described by connecting the parts. The worker model has one or several kinds of structure types. We call such types of structured data Model Worker. On the other side, workers basically have the same structure, but the detailed data belonging to a certain Model Worker in each instance (of human) is different. For example, an instance of a human body may have its own length of left and right arms and so on. The data are called Instance Worker. Instance Worker is an example of one of the Model Workers, and also it consists of own parts. However, each part does not have its own structure but only preserves scaling factors to the original model. Also there are some general rules for performing the instance works (e.g. instructions, directions, standards, etc.), whereas the instance work is different from each other. We call those Model Work.

All discussions are sumarized in a database schema by AIS diagram as shown in Figure 8. 
Table 3 Examples of Storing Human Body Structure by Database Functions

\begin{tabular}{lll}
\hline Database Function & $\begin{array}{c}\text { Domain } \\
\text { Value }\end{array}$ & $\begin{array}{c}\text { Range } \\
\text { Value }\end{array}$ \\
\hline Name.ModelWorkerPart.Parent.To.ModelWorkerPart.Name & LOIN & Base \\
\hline Name.ModelWorkerPart.RelativePosition.z & LOIN & $\mathbf{8 8 1 . 1}$ \\
\hline Name.ModelWorkerPart.RelativePosition. $\Theta_{x}$ & L_UPARM & -180.0 \\
\hline
\end{tabular}

Table 4 Examples of Storing Human Motion by Database Functions

\begin{tabular}{lll}
\hline Database Function & Domain Value & Range Value \\
\hline InstanceWorkerPart.Position. $\Theta_{x}$ & L_SHOULDER001 & $\{4.286,4.286, \ldots\}$ \\
\hline InstanceWorkerPart.Position. $\Theta_{x}$ & L_UPARM001 & $\{32.57,32.57, \ldots\}$ \\
\hline
\end{tabular}

\subsection{Description of Motions}

In Figure 8, the hierarchical structure of human parts is described by repetitive parent-child relationship on an entity type Model Worker Part. Also, a worker's motion is stored as a series of $\mathrm{X}, \mathrm{Y}$ and $\mathrm{Z}$ values. Those are represented by instance values of database functions.

Table 3 and Table 4 show examples of database functions and instances with domain and range values. Instances listed in Table 3 correspond to Table 1 (Human Body Structure).

And motion of a worker which is represented in Table 2 is stored like as shown Table 4.

In Table 4, the column "Range Value" holds sequences of values for each database function. Each value of the sequence represents a joint angle of a particular point of time. In short, a position of a part of a human body at a particular time is represented by the Cartesian Product of entity types Instance Worker Parts and Point of Time as shown in Figure 8.

\section{QUERY LANGUAGE AND SPATIO-TEMPORAL INQUIRY}

In order to derive arbitrary complex objects from a "flat" database, we have proposed a new data manipulation language - MMQL (Multimedia Query Language) (Arisawa, H. et al. 1996) defined on the AIS Data Model. MMQL is a functional query language and is based upon FP (Backus, J. 1978) as suggested by Backus - similar approach appears in FQL (Buneman, P. et al. 1982). However, FQL fails in handling large amounts of data because the operators are not suitable for implementing parallel computations and treating multimedia data are omitted. 


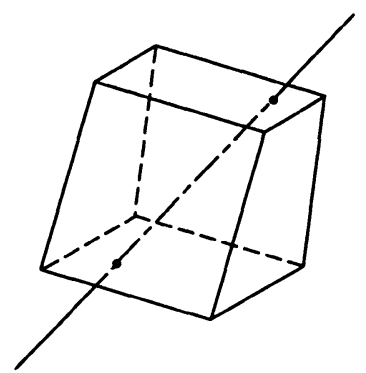

(a) through

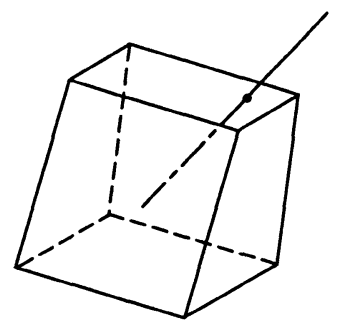

(b) stick

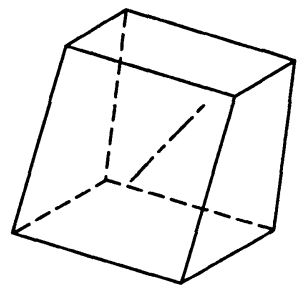

(c) inside

Figure 9 relation between a segment and a polygon object

\subsection{Spatial Relationship}

In order to retrieve $3 \mathrm{D}$ objects in a time-space, we developed the methodology calculating relative positions and temporal relationships between objects.

Descriptions of relative positions on two regions are discussed in GIS. Belussi, A. et al. (1997), Chan, P.P. et al. (1997) and Gueting et al. (1995) present regional constraints and predicates. Also Bimbo, A.D. et al. (1995) proposed a complete spatio-temporal logic in an iconic environment.

This paper proposes a function that can calculate the relative position in an arbitrary part of 3D objects involving a number of polygons. The function "relation(A, B)" calculates the relationship between the position of two regions $A$ and $B$ like shown in Figure 9. In this example, $A$ and $B$ represent a segment and a polygon object, respectively. All relationships are summarized in Table 5 similar to Allen's temporal logic (Allen, J.F. 1983) or Spatial SQL (Egenhofer, M.J. 1994). The function returns value "disjoint" in case its arguments are disjoint, or if not - the corresponding entry from Table 5. All the entries represent some kinds of "intersect." One region usually represents an object which is stored in the database and is generated in a phase of an inquiry. The other may be defined arbitrarily in the semantics of the inquiry by the user.

\subsection{Spatio-Temporal Query by MMQL}

A brief example of spatio-temporal inquiry is shown in Figure 10. The meaning of the inquiry is: "in a model work, show all the objects that intersect REGION." We assume that REGION is a result of another MMQL query, calculating the surface that includes particular worker's hands.

In general, similar to FP, in MMQL the composition between several descriptions is denoted by "o", and composed descriptions will be evaluated in turn. In the example, each description has an operator such as " $\alpha$ " or " $\beta$ ", names of a hierarchy of query result as "CG:" and functions such as "ap- 
Table 5 Relationship between two regions

\begin{tabular}{|c|c|c|c|c|c|c|}
\hline & point & line & segment & surface & a polygon & polygon object \\
\hline point & equal & onto & onto & onto & onto & inside \\
\hline line & include & $\begin{array}{l}\text { equal } \\
\text { cross }\end{array}$ & $\begin{array}{l}\text { include } \\
\text { cross } \\
\text { meet }\end{array}$ & $\begin{array}{l}\text { onto } \\
\text { cross }\end{array}$ & $\begin{array}{l}\text { divide } \\
\text { cross } \\
\text { meet }\end{array}$ & $\begin{array}{c}\text { meet } \\
\text { through }\end{array}$ \\
\hline segment & include & $\begin{array}{l}\text { onto } \\
\text { cross } \\
\text { meet }\end{array}$ & $\begin{array}{l}\text { equal } \\
\text { include } \\
\text { onto } \\
\text { overlap } \\
\text { meet } \\
\text { cross }\end{array}$ & $\begin{array}{l}\text { onto } \\
\text { cross } \\
\text { meet }\end{array}$ & $\begin{array}{c}\text { onto } \\
\text { stick } \\
\text { divide } \\
\text { meet } \\
\text { cross }\end{array}$ & $\begin{array}{c}\text { meet } \\
\text { through } \\
\text { stick } \\
\text { inside }\end{array}$ \\
\hline surface & include & $\begin{array}{l}\text { include } \\
\text { cross }\end{array}$ & $\begin{array}{l}\text { include } \\
\text { cross } \\
\text { meet }\end{array}$ & $\begin{array}{l}\text { equal } \\
\text { cross }\end{array}$ & $\begin{array}{c}\text { include } \\
\text { cross } \\
\text { meet }\end{array}$ & $\begin{array}{c}\text { meet } \\
\text { divide }\end{array}$ \\
\hline a polygon & include & $\begin{array}{l}\text { divided } \\
\text { meet } \\
\text { cross }\end{array}$ & $\begin{array}{l}\text { include } \\
\text { stuck } \\
\text { divided } \\
\text { meet } \\
\text { cross }\end{array}$ & $\begin{array}{l}\text { onto } \\
\text { cross } \\
\text { meet }\end{array}$ & $\begin{array}{l}\text { equal } \\
\text { include } \\
\text { onto } \\
\text { overlap } \\
\text { meet } \\
\text { cross }\end{array}$ & $\begin{array}{c}\text { meet } \\
\text { divide } \\
\text { stick } \\
\text { inside }\end{array}$ \\
\hline polygon object & contain & $\begin{array}{c}\text { meet } \\
\text { penetrated }\end{array}$ & $\begin{array}{c}\text { meet } \\
\text { penetrated } \\
\text { stuck } \\
\text { contain }\end{array}$ & $\begin{array}{c}\text { meet } \\
\text { divided }\end{array}$ & $\begin{array}{l}\text { meet } \\
\text { divided } \\
\text { stuck } \\
\text { contain }\end{array}$ & $\begin{array}{l}\text { equal } \\
\text { inside } \\
\text { contain } \\
\text { overlap } \\
\text { meet }\end{array}$ \\
\hline
\end{tabular}

ply(...)" or "relation(...)". The operator " $\alpha$ ", similar to the join operator in SQL, means apply to all operator in FP, and distributes successive functions to apply. On the other hand, the operator " $\beta$ ", similar to selection operator in SQL, means the restriction operator in FQL.

The function "relation" is shown in the example. It calculates the relationship between the position of two regions as described in the former subsection. When the user considers one region a surface which moves temporally, such inquiry is called "the query by space-time section" because the surface cuts the cyberspace. As a result of the query, objects which intersect the space-time section in practical instance of work are obtained.

Figure 11 shows a conceptual example of a space-time section in which a region is defined as a closed surface between both hands of a worker, and a hand drill is obtained as a result object of the query. Such an image is created by CWR following to query result.

As discussed above, MMQL provides powerful and flexible data retrieval. As a most interesting type of query we consider the reconstructing of a new world using CG data from the database. Generally, CG Data includes figures, polygons, textures, positions, light sources, view points, motions and so on. Thus, 3D CG data is huge and extremely complex. If we add the query in 
WORK : apply(ModelWork.InstanceWork)

$\circ \alpha</$ WORK $>$ [TIME : apply(InstanceWork.Frame.PointOfTime)

OBJ : apply(InstanceWork.InstanceWorker)]

$\circ \alpha</$ WORK/TIME $>$

[CG : calculatepolygon(PointofTime.Position.InstanceWorkerParts), REGION]

$\circ \alpha</$ WORK/TIME $>$ PHASE : relation(REGION, CG)

$\circ \beta</$ WORK $>$ (intersect in TIME/PHASE)

Figure 10 A Brief Example of Spatio-Temporal Query by MMQL (1)

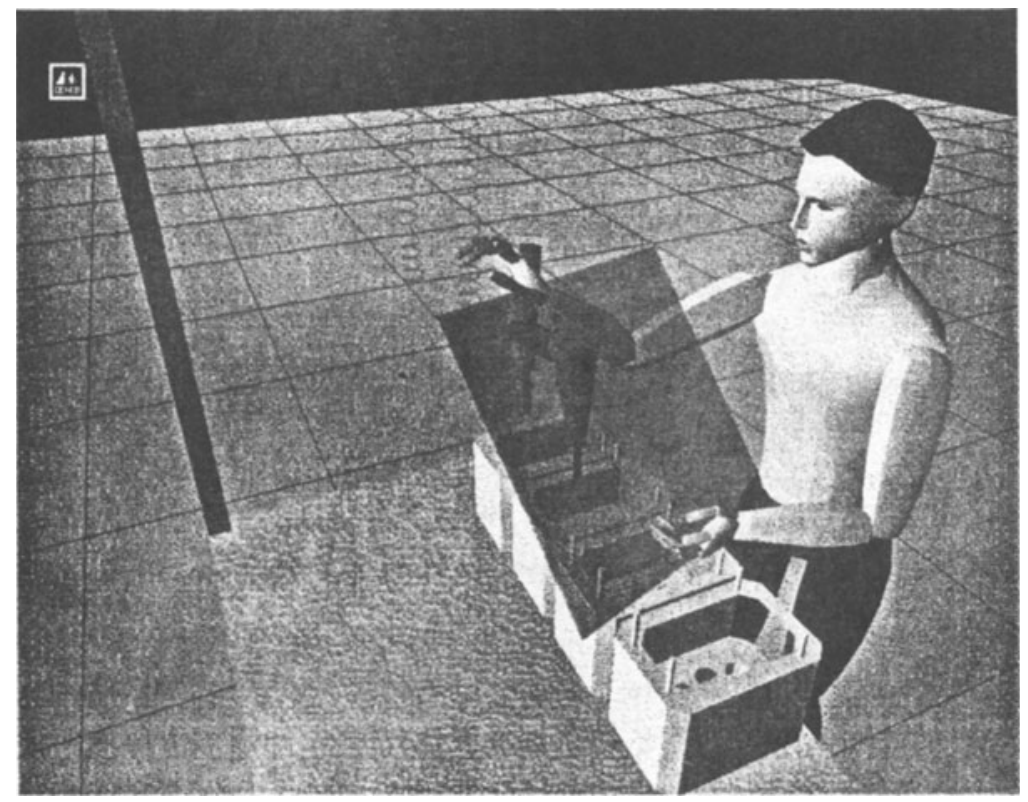

Figure 11 conceptual example of space-time section 


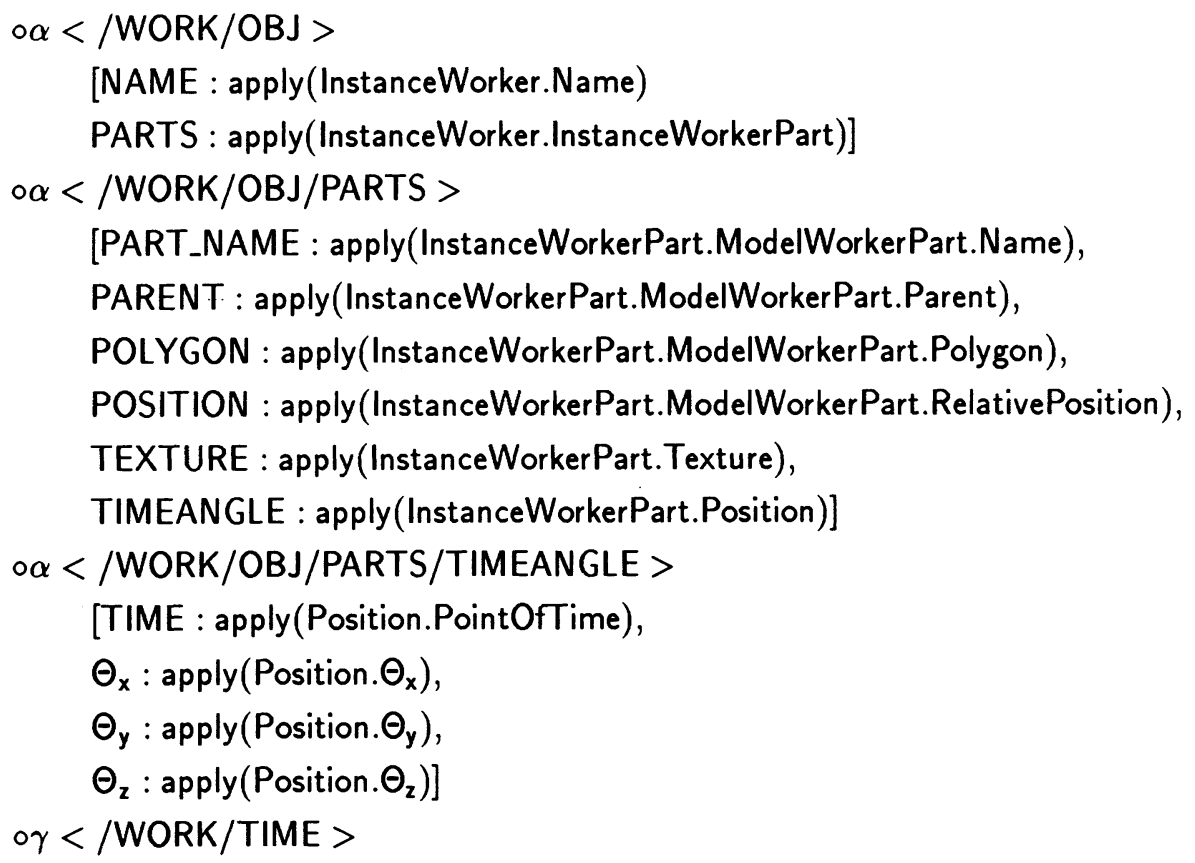

Figure 12 A Brief Example of Spatio-Temporal Query by MMQL (2)

Figure 12 to the previous query (Figure 10), the result will be a new query: "create a new "world" that includes only the retrieved objects."

On the other hand, work data in RWDB has a flat structure. Therefore, a query has to derive structured data from the flat DB structure, and to reconstruct the real world into a virtual world. An outlook of the query result is shown in Figure 13. We call this structured data OE (Object Expression). This OE contains all retrieved spatio-temporal data, thus it allows to perform CG and/or VR simulations. CG Work Simulation is implemented by using OE on CWR.

\section{CONCLUDING REMARKS}

This paper reported the concept of Real World Database, and modelling of human and its work based on functional data model. Also spatio-temporal inquiry that is designed by using MMQL has been described. Such an approach would be beneficial to manufacturing process design, rehabilitation in medical areas, etc. The prototype system of RWDB and Info-Ergonomics simulation has been developed by the authors. In the current system, RWC is embodied 


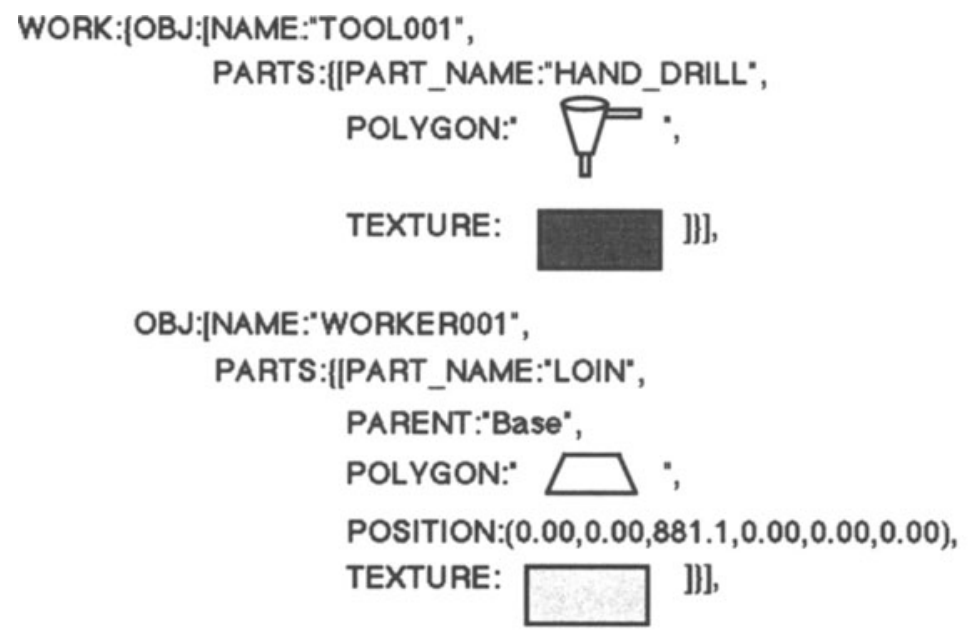

TIMEANGLE:[[TIME:0, $\theta \mathrm{x}: 0.00, \theta \mathrm{y}: 0.00, \theta \mathrm{z}: 0.00]$,

[TIME:0.50, $\theta$ x:0.00, $\theta$ y:0.00, $\theta \mathrm{z}: 0.00]$,

[TIME:1.00, $\theta$ x:0.00, $\theta$ y:0.00, $\theta \mathrm{z}: 0.00], \ldots$.$] ],$

[PART_NAME:L_SHOULDER',

\section{PARENT:"CHEST",}

POLYGON:" ',

POSITION:(0.00,65.00,207.20,0.00,0.00,0.00),

TEXTURE:

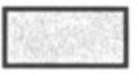

]l],

TIMEANGLE:[[TIME:0, $\theta$ x:4.286, $\theta$ y:9.249, $\theta$ z:0.00],

[TIME:0.50, $\theta \times: 5.286, \theta$ y:4.249, $\theta \mathrm{z}: 0.00]$ ],

[TIME:1.00, $\theta x: 5.846, \theta y: 4.809, \theta z: 0.00], \ldots .$.$] ],$

[PART_NAME:"L_UPARM",

PARENT:"L_SHOULDER",

POLYGON:" \] ',

POSITION:(0.00,1044.80,0.00,-180.00,0.00,0.00),

TEXTURE: †且

TIMEANGLE:([TIME:0, $\theta \times 32.57, \theta$ y:0.00, $\theta \quad z: 0.00]$,

[TIME:0.50, $\theta \times 32.57, \theta y: 0.00, \theta z: 0.00]$ ],

[TIME:1.00, $\theta \times 32.57, \theta y: 0.00, \theta z: 0.00], \ldots]$.$] ,$

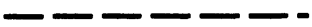

Figure 13 An example of a query result 
by VGA cameras and Digital Video Disk Array (DVDA). RWM and MMDB are implemented on work stations, and CWR on a graphic work station with the stereo visualization function. Future work should focus on the optimization of query processing and the further development of the simulation system.

\section{ACKNOWLEDGEMENT}

This work is partly supported by the Grant-in-Aid for Scientific Research of the Ministry of Education, Science and Culture of Japan. Also, a part of this research is supported by the IMS project Japan.

\section{REFERENCES}

Allen, J.F. (1983) Maintaining Knowledge about Temporal Intervals, Comm. $A C M, 26$, No.11, pp.832-843.

Arisawa, H. (1996) Considerations on Real World Data Modelling, Technical Report of IEICE, The Institute of Electronics, Infomation and Communication, DE96-4, 19-24(in Japanese).

Arisawa, H. and Imai, S. (1996) Working Simulation based on Info-Ergonomics and Multimedia Database Concept Design, Japan-U.S.A. Symposium on Flexible Automation.

Arisawa, H., Tomii, T. and Salev, K. (1996) Design of Multimedia Database and a Query Language for Video Image Data, Proc. International Conference on Multimedia Computing and Systems, IEEE, Hiroshima, Japan, June 1996, 462-7.

Arisawa, H., Tomii, T., Yui, H. and Ishikawa, H. (1995) Data Model and Architecture of Multimedia Database for Engineering Applications, IEICE Trans. Inf. \& Syst., The Institute of Electronics, Infomation and Communication, E78-D, No.11, 1362-8.

Backus, J. (1978) Can Programming Be Liberated from the von Neumann Style? A Functional Style and Its Algebra of Programs, Comm. ACM, 21, No.8, 613-641.

Belussi, A., Bertino, E. and Catania, B. (1997) Manipulating Spatial Data in Constraint Databases, Proc. of 5th International Symposium on Spatial Databases (SSD'97), 115-141, Berlin, Germany, July 1997, Lecture Notes in Computer Science, 1262, Springer-Verlag.

Bimbo, A.D., Vicario, E. and Zingoni, D. (1995) Symbolic Description and Visual Querying of Image Sequences Using Spatio-Temporal Logic, IEEE Trans. on Knowledge and Data Engineering, 7, No.4, 609-622.

Buneman, P., Frankel, R.E. and Nikhil, R. (1982) An Implementation Technique for Database Query Languages, ACM Trans. on Database Systems, 7, No.2, 164-186.

Chan, E.P.F. and Ng, J.N.H. (1997) A General and Efficient Implementation 
of Geometric Operations and Predicates, Proc. of 5th International Symposium on Spatial Databases (SSD'97), 69-93, Berlin, Germany, July 1997, Lecture Notes in Computer Science, 1262, Springer-Verlag. Chen, P.P. (1976) The Entity-Relationship Model Toward a Unified View of Data, ACM Trans. on Database Systems, 1, No.1, 1-49.

Egenhofer, M.J. (1994) Spatial SQL: A Query and Presentation Language, IEEE Trans. on Knowledge and Data Engineering, 6, No.1, 86-95.

Gueting, R.H. and Schneider, M. (1995) Realm-Based Spatial Data Types: The ROSE Algebra, VLDB Journal, 4, 243-286.

Hirose, M. (1997) Image-Based Virtural World Generation, IEEE Multimedia, Jan-Mar 1997, 27-33.

Kanade, T., Rander, P. and Narayanan, P.J. (1997) Virtualized Reality: Constructing Virtual Worlds from Real Scenes, IEEE Multimedia, Jan-Mar 1997, 34-47.

\section{BIOGRAPHY}

Takashi Tomii graduated Division of Electrical and Computer Engineering, Faculty of Engineering, Yokohama National University in 1994, and received the M.E. degrees in 1996. He is now Ph.D candidate student. His current research interests include the next generation database systems, multimedia databases, spatio-temporal modelling, query language and their implementation.

Kiril Salev received a M.Sc. degree in Computer Science from the Faculty of Mathematics and Informatics of Sofia University "St. Kliment Ohridski" in 1992. At present he is a Ph.D. student with the Graduate School of Engineering of Yokohama National University.

Sayaka Imai graduated Division of Electrical and Computer Engineering, Faculty of Engineering, Yokohama National University in 1995. She is now Ph.D candidate student.

Hiroshi Arisawa was born in Tokyo, Japan, on Sept. 26, 1948. He received B.S. from the University of Tokyo in 1972 and Ph.D. degree (Dr. Eng) from Kyoto University in 1986 respectively. He was engaged in Fujitsu Ltd. from 1973 to 1974 . In 1975, he joined to Yokohama National University. Now he is a professor in the Division of Electrical and Computer Engineering. His research interest covers: theory of database design and database systems, multimedia databases and video-data analysis. From 1986 to 1989, he was the chief of editorial WG of "Journal of Information Processing." He was also a visiting associate professor of Oregon State University from February to December in 1991. 